\title{
A MULTIFUNCTION GENERALIZATION OF GALE'S ASCOLI THEOREM
}

\author{
GEOFFREY FOX and PEDRO MORALES
}

(Received 16 October 1972; revised 25 June 1973)

Communicated by E. Strzelecki

\section{Introduction}

Our purpose is to improve the Gale-type multifunction Ascoli theorem of Mancuso (1971; page 470). This latter supposes the range space to be normal and Hausdorff, and therefore does not contain Gale's theorem (1950; page 304). To obtain a multifunction theorem containing Gale's theorem (also Mancuso's theorem), we return to Gale's essential hypotheses. Thus, we assume the regularity of the range space in the sufficiency direction, and, in the necessity direction, we assume the domain to be a $k$-space and the range to be a regular Hausdorff space. We dispense with the "point-like" condition imposed by Mancuso. Unexplained terminology and notation is that of Mancuso's paper.

\section{Multifunctions with values in a regular space}

Let $X, Y$ be topological spaces. A multifunction $f: X \rightarrow Y$ is lower (upper) semi-continuous if $f^{-}(U)\left(f^{+}(U)\right)$ is open in $X$ whenever $U$ is open in Y. Consequently, $f$ is continuous if and only if $f$ is both lower and upper semi-continuous.

LeMma 1. Let $f: X \rightarrow Y$ be a multifunction on a topological space $X$ to a regular space $Y$. Then $f$ is lower semi-continuous if, for each $x \in X$ and each open set $U$ of $Y$ such that $f x \cap U \neq \varnothing$, there exists a neighbourhood $N$ of $x$ such that $f z \cap \bar{U} \neq \varnothing$ for all $z \in N$.

Proof. Let $G$ be an open set of $Y$ and let $x \in f^{-}(G)$. Then $f x \cap G \neq \varnothing$. Let $y \in f x \cap G$. By the regularity, there is an open set $U$ in $Y$ such that $y \in U \subset \bar{U} \subset G$. Since $f x \cap U \neq \varnothing$, there exists, by hypothesis, a neighbourhood $N$ of $x$ such that, if $z \in N, f z \cap \bar{U} \neq \varnothing$. So $f z \cap G \neq \varnothing$, that is, $z \in f^{-}(G)$.

LeMma 2. Let $f: X \rightarrow Y$ be a point-compact multifunction on a topo 
logical space $X$ to a regular space $Y$. Then $f$ is upper semi-continuous if, for each $x \in X$ and each open set $U$ of $Y$ such that $f x \subset U$, there exists a neighbourhood $N$ of $x$ such that $f z \subset \bar{U}$ for all $z \in N$.

Proof. Let $G$ be an open set of $Y$ and let $x \in f^{+}(G)$. Then $f x \subset G$. Since $f x$ is compact and $Y$ is regular, there is an open set $U$ in $Y$ such that $f x \subset U \subset \bar{U} \subset G$. By hypothesis, there exists a neighbourhood $N$ of $x$ such that if $z \in N$, then $f z \subset \bar{U}$. So $f z \subset G$, that is, $z \in f^{+}(G)$.

The following lemma generalizes Lemma 2.4 of Mancuso (1971; page 467), and will be crucial in the proof of our Ascoli theorem:

LeMma 3. Let $f: X \rightarrow Y$ be a continuous multifunction on a topological space $X$ to a regular space $Y$. If $f x \cap U \neq \varnothing$ for some open set $U$ in $Y$, there exists a neighbourhood $V$ of $x$ such that $f z \cap U \neq \varnothing$ for all $z \in \bar{V}$.

Proof. Let $y \in f x \cap U$. By the regularity there is an open set $W$ in $Y$ such that $y \in W \subset \bar{W} \subset U$. Since $f$ is lower semi-continuous, there exists a neighbourhood $V$ of $x$ such that $V \subset f^{-}(W)$. Let $z \in \bar{V}$ and suppose that $f z \cap U=\varnothing$. Then $f z \cap \bar{W}=\varnothing$ so that $f z \subset Y-W$. Since $f$ is upper semi-continuous, there is a neighbourhood $H$ of $z$ such that $H \subset f^{+}(Y-\bar{W})$. Since $z \in \bar{V}$, there is $v \in H \cap V$. Then $f v \subset Y-\bar{W}$ and therefore $f v \cap W=\varnothing$. This is a contradiction, because $v \in V \subset f^{-}(W)$.

\section{Condition (G)}

Let $X, Y$ be topological spaces and let $F \subset Y^{m X}$. The set of subsets of $F$ of the form $\{f: f(K) \subset U$ and $f x \cap V \neq \varnothing$ for all $x \in K\}$, where $K$ is a compact subset of $X$ and $U, V$ are open in $Y$, is an open subbase for the compact open topology $\tau_{c}$ on $F$ [7, page 47]. It is clear that $\tau_{c}$ is larger than the pointwise topology $\tau_{p}$ on $F$. The family $F$ is called collectively continuous if $U_{f \in \mathrm{F}} f^{+}(B)$ and $\cup_{f \in \mathrm{F}} f^{-}(B)$ are closed in $X$ whenever $B$ is closed in $Y$, or, equivalently, $\cap_{f \in \mathrm{F}} f^{+}(B)$ and $\cap_{f \in \mathrm{F}} f^{-}(B)$ are open in $X$ whenever $B$ is open in $Y$ (Mancuso (1971; page 469)). We say that $F$ satisfies the condition $(G)$ if each closed subset of $\left(F, \tau_{c}\right)$ is collectively continuous.

LEMMA 4. Let $F$ be a family of point-compact multifunctions on a topological space $X$ to a regular space $Y$. If $F$ satisfies $(G)$, then the members of the $\tau_{p}$-closure $\bar{F}$ of $F$, in the set of point-compact multifunctions of $Y^{m X}$, are continuous.

Proof. Let $f \in F$. To show that $f$ is lower semi-continuous, suppose $f x \cap V \neq \varnothing$, where $V$ is open in $Y$. By Lemma 1, it suffices to show that there exists a neighbourhood $N$ of $x$ such that $f z \cap \bar{V} \neq \varnothing$ for all $z \in N$. Let $y \in f x \cap V$ and let $G$ be an open set in $Y$ such that $y \in G \subset \bar{G} \subset V$. Then $F_{0}=\{h: h \in F$ and $h x \cap \bar{G} \neq \varnothing\}$ is closed in $\left(F, \tau_{c}\right)$. Since $F$ satisfies $(G), F_{0}$ is 
collectively continuous. Then $N=\bigcap_{h \in F_{0}} h^{-}(V)$ is an open set in $X$ containing $x$. We claim that $z \in N$ implies $f z \cap \bar{V} \neq \varnothing$. In fact, suppose that $f z \cap \bar{V}=\varnothing$. Then $f z \subset Y-\vec{V}$, and, since $f z$ is compact, there exists an open set $W$ such that $f z \subset W \subset \bar{W} \subset Y-\bar{V}$. Since $M=\left\{g: g \in Y^{m X}, g\right.$ is point-compact, $g z \subset W$ and $g x \cap G \neq \varnothing\}$ is a $\tau_{p}$ neighbourhood of $f$, there exists $f^{\prime} \in F \cap M$. Then $f^{\prime} \in F_{0}$, and, since $z \in N, f^{\prime} z \cap V \neq \varnothing$. This is a contradiction, because $f^{\prime} z \subset W$ and $W \cap V=\varnothing$.

To show that $f$ is upper semi-continuous, suppose $f x \subset V$, where $V$ is open in $Y$. By Lemma 2, it suffices to show that there exists a neighbourhood $N$ of $x$ such that $f z \subset \bar{\nabla}$ for all $z \in N$. Since $f x$ is compact there exists an open set $G$ in $Y$ such that $f x \subset G \subset \bar{G} \subset V$. Then $F_{0}=\{h: h \in F$ and $h x \subset \bar{G}\}$ is closed in $\left(F, \tau_{c}\right)$. Since $F$ satisfies $(G), F_{0}$ is collectively continuous. Then $N=\bigcap_{h \in \mathrm{F}_{0}} h^{+}(V)$ is an open set in $X$ containing $x$. We claim that $z \in N$ implies $f z \subset \bar{V}$. In fact, let $z \in N, y \in f z$ and let $W$ be any neighbourhood of $y$. Since $M=\left\{g: g \in Y^{m X}\right.$, $g$ is point-compact, $g x \subset G$ and $g z \cap W \neq \varnothing\}$ is a $\tau_{p}$-neighbourhood of $f$, there exists $f^{\prime} \in M \cap F$. Then $f^{\prime} z \cap W \neq \varnothing$ and $f^{\prime} \in F_{0}$, so, because $z \in N$, we have $f^{\prime} z \subset V$. Thus $W \cap V \neq \varnothing$, so $y \in \bar{V}$. Since $y$ is an arbitrary point of $f z, f z \subset \bar{V}$.

COROLlary 1. Let $F$ be a family of point-compact multifunctions on a topological space $X$ to a regular space $Y$. If $F$ satisfies $(G)$, then the members of $F$ are continuous.

Corollary 2. Let $F$ be a family of functions on a topological space $X$ to a regular space $Y$. If $F$ satisfies $(G)$, then the members of the $\tau_{p}$-closure of $F$ in $Y^{X}$ are continuous.

LEMMA 5. Let $F$ be a family of point-compact multifunctions on a topological space $X$ to a regular space $Y$. If $F$ satisfies $(G)$, then the $\tau_{p}$-closure and the $\tau_{c}$-closure of $F$, in the set of point-compact multifunctions of $Y^{m X}$, are identical.

Proof. Let $F, \tilde{F}$ denote the $\tau_{p}$-closure, the $\tau_{c}$-closure of $F$, respectively, in the set of point compact multifunctions of $Y^{m X}$. It must be shown that $f_{0} \in \bar{F}$ implies $f_{0} \in \tilde{F}$. It will suffice to show that every $\tau_{c}$-neighbourhood of $f_{0}$ of the form $\bigcap_{i=1}^{n}\left\{f: f \in Y^{m X}, f\right.$ is point-compact, $f\left(K_{i}\right) \subset U_{i}$ and $f x \cap U_{i}^{\prime} \neq \varnothing$ for all $\left.x \in K_{i}\right\}$, where $K_{i} \subset X$ is compact and $U_{i}, U_{i}^{\prime} \subset Y$ are open $(i=1, \cdots, n)$ intersects $F$. Since $f_{0}$ is continuous (Lemma 4 ) and point-compact, $f_{0}\left(K_{i}\right)$ is a compact subset of $U_{i}(i=1, \cdots, n)$ (Berge (1965; page 110)). Then there exists, for each $i=1, \cdots, n$, an open set $V_{i}$ in $Y$ such that $f_{0}\left(K_{i}\right) \subset V_{i} \subset V_{i} \subset U_{i}$. Since $f_{0} x \cap U_{i}^{\prime} \neq \varnothing$ for all $x \in K_{i}(i=1, \cdots, n)$, by the Lemma 1 of Smithson (1971; page 47), there exists an open set $V_{i}^{\prime}$ in $Y$ such that $\bar{V}_{i}^{\prime} \subset U_{i}^{\prime}$ and $f_{0} x \cap V_{i}^{\prime} \neq \varnothing$ for all $x \in K_{i}$. Choose a fixed index $i$. For $x \in K_{i}$ let $H_{x}=\left\{f: f \in Y^{m x}, f\right.$ is pointcompact, $f x \subset \bar{V}_{i}$ and $\left.f x \cap \bar{V}_{i}^{\prime} \neq \varnothing\right\}$. Then $H_{x}$ is a closed $\tau_{p}$-neighbourhood 
of $f_{0}$, and therefore $F_{x}=H_{x} \cap F$ is a non-empty closed subset of $\left(F, \tau_{c}\right)$. Since $F$ satisfies $(G), F_{x}$ is collectively continuous. Then $N_{x}=\bigcap_{f \in \mathbf{F} .} f^{+}\left(U_{i}\right) \cap$ $\bigcap_{f \in \mathbf{F}_{x}} f^{-}\left(U_{i}^{\prime}\right)$ is open in $X$, and contains $x$, because $f \in F_{\lambda}$ implies $f x \subset \bar{V}_{i} \subset U_{i}$ and $f x \cap U_{i}^{\prime} \supset f x \cap \bar{V}_{i} \neq \varnothing$. Since $K_{i}$ is compact, there is a finite sequence $\left\{x_{j}^{i}\right\}_{1 \leqq j \leqq k_{i}}$ in $K_{i}$ and corresponding neighbourhoods $H x_{j}^{i}, N x_{j}^{i}$ of $f_{0}, x_{j}^{i}$, respectively, such that $K_{i} \subset \bigcup_{j=1}^{k_{i}} N x_{j}^{i}$. Then $M=\cap_{i=1}^{n} \cap_{j=1}^{k_{i}} H_{x j}$ is a $\tau_{p}$-neighbourhood of $f_{0}$. Hence there exists $f^{\prime} \in M \cap F$. The proof will be complete if we show that, for any $i=1, \cdots, n, f^{\prime}\left(K_{i}\right) \subset U_{i}$ and $f^{\prime} x \cap U_{i}^{\prime} \neq \varnothing$ for all, $x \in K_{i}$. Let $x \in K_{i}$. There exists $j\left(1 \leqq j \leqq k_{i}\right)$ such that $x \in N x_{j}^{\prime} \subset \bigcap_{f \in F x_{j}^{\prime}} f^{-}\left(U_{i}^{\prime}\right)$ and therefore $f x \cap U_{i}^{\prime} \neq \varnothing$ for all $f \in F_{x j}^{i}$. Since $f^{\prime} \in M \cap F \subset H_{x_{j}^{\prime}} \cap F=F_{x_{j}}$ we have $f^{\prime} x \cap U_{i}^{\prime} \neq \varnothing$. On the other hand, $x \in N x_{j}^{t} \subset \bigcap_{f \in F x_{j}{ }^{t}} f^{+}\left(U_{i}\right)$. Then $f x \subset U_{i}$ for all $f \in F x_{j}^{\prime}$, and, in particular, $f^{\prime} x \subset U_{i}$. Since $x$ is an arbitrary point of $K_{i}, f^{\prime}\left(K_{i}\right) \subset U_{i}$.

COROLlaRY 3. Let $F$ be a family of point-compact multifunctions on a topological space $X$ to a regular space $Y$. If $F$ satisfies $(G)$, then $\tau_{p}=\tau_{c}$ on $F$.

Proof. It suffices to show that, on $F, \tau_{p}$ is larger than $\tau_{c}$. Let $H$ be closed in $\left(F, \tau_{c}\right)$. Obviously $H$ satisfies $(G)$, so $\bar{H}=\tilde{H}$. Hence $H=\tilde{H} \cap F=\bar{H} \cap F$ is closed in $\left(F, \tau_{p}\right)$.

\section{Ascoli theorem}

A subset $E$ of a topological space $X$ is $k$-closed if $E \cap K$ is closed in $K$ for every compact subset $K$ of $X$. A topological space is a $k$-space if every $k$-closed subset is closed Cohen (1954; page 79). Locally compact spaces and spaces satisfying the first countability axiom are familiar examples of $k$-spaces.

LEMMA 6. Let $F_{0}$ be the family of all point-compact multifunctions on a set $X$ to a Hausdorff space $Y$. Then $\left(F_{0}, \tau_{p}\right)$ is Hausdorff.

Proof. Let $f, g$ be distinct members of $F_{0}$; we may suppose a $y \in f x-g x$ for some $x \in X$ and some $y \in Y$. We can then construct disjoint neighbourhoods of $y, g x$, from which we define disjoint neighbourhoods of $f, g$.

Let $F_{0}$ be the family of all point-compact multifunctions on a set $X$ to a topological space $Y$, and let $F \subset F_{0}$. We will say that a subset $B$ of $F_{0}$ covers $F$ if $\bar{F} \subset B$, where $F$ denotes the $\tau_{p}$-closure of $F$ in $F_{0}$. If $X, Y$ are topological spaces, the symbol $\mathscr{C}(X, Y)(C(X, Y))$ will denote the subfamily consisting of all continuous multifunctions (continuous functions) of $F_{0}$. If $Y$ is regular and $F \subset F_{0}$ satisfies $(G)$, then $\mathscr{C}(X, Y)$ covers $F$ (Lemma 4). If, further, $F$ consists of functions, then $C(X, Y)$ covers $F$ (Corollary 2 ).

The following lemma appears as Corollary 3 in Fox and Morales (to appear):

Lemma 7. Let $\left\{X_{a}\right\}_{a \in A}$ be a non-empty family of compact spaces. The set of all point-compact multifunctions of $P\left\{X_{a}: a \in A\right\}$ is $\tau_{p}$-compact. 
Let $\left\{X_{a}\right\}_{a \in A}$ be a family of non-empty sets and let $F \subset P\left\{X_{a}: a \in A\right\}$. For $a \in A$, we write $F[a]=\bigcup_{f \in \mathrm{F}} f a$.

LEMMA 8. Let $\left\{X_{a}\right\}_{a \in A}$ be a non-empty family of topological spaces. If $F \subset P\left\{X_{a}: a \in A\right\}$ then $\bar{F} \subset P(\overline{\{[a]}: a \in A\}$, where $\bar{F}$ denotes the $\tau_{p}$-closure of $F$ in $P\left\{X_{a}: a \in A\right\}$.

Proof. Let $f \in F$. Let $a \in A$ be arbitrary, let $y \in f a$, and let $V$ be an arbitrary neighbourhood of $y$. Since $M=\left\{h: h \in P\left\{X_{a}: a \in A\right\}\right.$ and $\left.h a \cap V \neq \varnothing\right\}$ is a $\tau_{p}$-neighbourhood of $f$, there exists $f^{\prime} \in M \cap F$. Then $f^{\prime} a \cap V \neq \varnothing$ and $f^{\prime} a \subset F[a]$, and therefore $F[a] \cap V \neq \varnothing$. So $y \in \overline{F[a]}$. Since $y$ is an arbitrary point of $f a$, we have $f a \subset \overline{F[a]}$.

THEOREM. Let $F$ be a family of point-compact continuous multifunctions on a topological space $X$ to a regular space $Y$, and let $B$ be a family of pointcompact multifunctions of $Y^{m X}$ which covers $F$. The conditions

(a) $F$ is closed in $\left(B, \tau_{c}\right)$,

(b) $F[x]$ is compact for all $x \in X$, and

(c) F satisfies $(G)$,

are sufficient for the $\tau_{c}$-compactness of $F$. If, further, $X$ is a $k$-space and $Y$ is Hausdorff, then the condition (a), (b) and (c) are necessary for the $\tau_{c}$-compactness of $F$.

Proor. Sufficiency. Let $F_{0}, F_{0}^{\prime}$ be the sets of all point-compact multifunctions of $Y^{m X}, P\{\overline{F[x]}: x \in X\}$, respectively. If $\bar{F}$ denotes the $\tau_{-p}$ closure of $F$ in $F_{0}$, Lemma 8 implies $F \subset F_{0}^{\prime} \subset F_{0}$. Since $Y$ is regular, (b) implies the compactness of $\overline{F[x]}$ for all $x \in X$. Then, by Lemma $7, F_{0}^{\prime}$ is $\tau_{p}$-compact, and so $\bar{F}$ is $\tau_{p}$-compact. Let $\tilde{F}$ denote the $\tau_{c}$-closure of $F$ in $F_{0}$. By Lemma $5,(c)$ implies $\tilde{F}=\bar{F}$. But $F \subset B$ and $(a)$ implies $F=\tilde{F} \cap B$, thus $F=F$ is $\tau_{p}$-compact. Since (c) implies $\left(F, \tau_{p}\right)=\left(F, \tau_{c}\right)$ (Corollary 3$), F$ is $\tau_{c}$-compact.

Necessity. Since $\left(B, \tau_{c}\right)$ is Hausdorff (Lemma 6$), F$ is closed in $\left(B, \tau_{c}\right)$. Since the projections $p r_{x}$ are point-compact and continuous, the $F[x]$ are compact. To prove that $F$ satisfies $(G)$ it will suffice to show that $F$ is collectively continuous. Since $X$ is a $k$-space, it will suffice to show that, if $W$ is closed in $Y$, then $\bigcup_{f \in \mathrm{F}} f^{+}(W)$ and $\bigcup_{f \in \mathrm{F}} f^{-}(W)$ are $k$-closed. Let $K$ be an arbitrary compact subset of $X$ and write $S=\bigcup_{f \in \mathrm{F}} f^{+}(W) \cap K, S^{\prime}=\bigcup_{f \in \mathrm{F}} f^{-}(W) \cap K$.

We will show that if $x \in K-S$, then $x \notin \bar{S} \cap K$. We have $f x \cap(Y-W) \neq \varnothing$ for all $f \in F$. Let $f \in F$. By Lemma 3, there exists a neighbourhood $N_{f}$ of $x$ such that $f z \cap(Y-W) \neq \varnothing$ for all $z \in \bar{N}_{f}$. Then $K_{f}=\bar{N}_{f} \cap K$ is compact, and therefore $M_{f}=\left\{h: h \in F\right.$ and $h z \cap(Y-W) \neq \varnothing$ for all $\left.z \in K_{f}\right\}$ is open in $\left(F, \tau_{c}\right)$ and contains $f$. Since $F$ is compact, there is a finite sequence $\left\{f_{i}\right\}_{1 \leqq i \leqq n}$ in $F$ such that $F \subset \bigcup_{i=1}^{n} M_{f_{i}}$. Let $K^{*}=\bigcap_{i=1}^{n} K_{f_{i}}$. Then, if $f \in F, f z \cap(Y-W) \neq \varnothing$ for all $z \in K^{*}$, and therefore $K^{*} \cap\left(\bigcup_{f \in \mathrm{F}} f^{+}(W)\right)=\varnothing$. On the other hand, 
$N^{*}=\bigcap_{i=1}^{n} N_{f_{i}}$ is a neighbourhood of $x$ such that $N^{*} \cap K \subset K^{*}$. Then $N^{*} \cap S=\varnothing$, proving the assertion.

We will show that if $x \in K-S^{\prime}$, then $x \notin \bar{S}^{\prime} \cap K$. We have $f x \subset Y-W$ for all $f \in F$. Let $f \in F$. There exists an open set $V_{f}$ in $Y$ such that $f x \subset V_{f} \subset \bar{V}_{f}$ $\subset Y-W$, and there exists a neighbourhood $N_{f}$ of $x$ such that $f\left(N_{f}\right) \subset V_{f}$. Then $\overline{f\left(N_{f}\right)} \subset Y-W$. Let $K_{f}=\bar{N}_{f} \cap K$. Then $K_{f}$ is compact, and therefore $M_{f}=\left\{h: h \in F\right.$ and $\left.h\left(K_{f}\right) \subset Y-W\right\}$ is open in $\left(F, \tau_{c}\right)$. Since $f\left(\bar{N}_{f}\right) \subset \overline{f\left(N_{f}\right)}$ Ponomarev (1960; page 120), $f \in M_{f}$. There is a finite sequence $\left\{f_{i}\right\}_{1 \leqq i \leqq n}$ in $F$ such that $F \subset \bigcup_{i=1}^{n} M_{f_{i}}$. Let $K^{*}=\bigcap_{i=1}^{n} K_{f i}$. Then, if $f \in F, f\left(K^{*}\right) \subset Y-W$, and therefore $K^{*} \cap\left(\bigcup_{f \in \mathrm{F}} f^{-}(W)\right)=\varnothing$. Let $N^{*}=\bigcap_{i=1}^{n} N_{f i}$. Then $N^{*}$ is a neighbourhood of $x$ such that $N^{*} \cap K \subset K^{*}$; therefore $N^{*} \cap S^{\prime}=\varnothing$, and the proof is complete.

COROLlary 4. Let $F$ be a family of point-compact continuous multifunctions on a topological space $X$ to a regular space $Y$. The conditions

(a) $F$ is closed in $\left(\mathscr{C}(X, Y), \tau_{c}\right)$,

(b) $F[x]$ is compact for all $x \in X$, and

(c) F satisfies $(G)$,

are sufficient for the $\tau_{c}$-compactness of $F$. If, further, $X$ is a $k$-space and $Y$ is Hausdorff, then the conditions (a), (b) and (c) are necessary for the $\tau_{c}$-compactness of $F$.

COROLLARY 5. Let $F$ be a family of continuous functions on a topological space $X$ to a regular space $Y$. The conditions

(a) $F$ is closed in $\left(C(X, Y), \tau_{c}\right)$,

(b) $F[x]$ is compact for all $x \in X$, and

(c) $F$ satisfies $(G)$,

are sufficient for the $\tau_{c}$-compactness of $F$. If, further, $X$ is a $k$-space and $Y$ is Hausdorff, then the conditions (a), (b) and (c) are necessary for the $\tau_{c}$-compactness of $F$.

Remarks 1. Corollary 5 is the essential Theorem 1 of Gale (1950; page 304).

2. The point-like condition of Mancuso played two roles, one of which we have by-passed by working entirely in the space of point-compact multifunctions. The second role was to assure the non-emptiness of the sets $F_{0}$ of our Lemma 4 (logically superfluous). However, without evoking the point-like condition, we may if we wish, prove these sets $F_{0}$ non-empty. For example, in the first case, we consider $M=\left\{g: g \in Y^{m X}, g\right.$ is point-compact and $\left.g x \cap G \neq \varnothing\right\}$, which is $a \tau_{p}$-neighbourhood of $f$, so there exists $f^{\prime} \in F \cap M \subset F_{0}$. 


\section{References}

C. Berge (1965), Topological Spaces, (The MacMillan Company, New York (1965).)

D. E. Cohen (1954), 'Spaces with weak topology', Quart. J. Math. Oxford Ser. (2) 5, 77 -80.

G. Fox and P. Morales (to appear), 'A general Tychonoff theorem for multifunctions', Canad. Math. Bull.

D. Gale (1950), 'Compact sets of functions and functions rings', Proc. Amer. Math. Soc. 1, 303-308.

V. J. Mancuso (1971), 'An Ascoli theorem for multi-valued functions', J. Austral. Math. Soc. $12,466-472$.

V. I. Ponomarev (1960), 'Properties of topological spaces preserved under multi-valued continuous mappings', Math. Sb. 5 (N.S.) 51 (93), 515-536 = Amer. Math. Soc. Transl. Ser (2) 38 (1968), 119-140.

R. E. Smithson (1971), 'Topologies on sets of relations', J. Math. Natur. Sci. and Math. (Lahore) 11, 43-50.

\section{Université de Montréal}

Montréal, Québec

Canada 